\title{
Comparison of Charged Derivatives for High Energy Collision-Induced Dissociation Tandem Mass Spectrometry
}

\author{
Joseph Zaia* and Klaus Biemann \\ Department of Chemistry, Massachusetts Institute of Technology, Cambridge, Massachusetts, USA
}

\begin{abstract}
Fixed-charge derivatives have been used to direct the fragmentation pattern of high energy collision-induced dissociation tandem mass spectra for several years. It has been noted that a fixed-charge placed at a terminus of a peptide will simplify the pattern of fragment ions that are produced in collision-induced dissociation. Trimethylammoniumacetyl, dimethyloctylammoniumacetyl, and triphenylphosphoniumethyl derivatives have been cited in the literature for this purpose and many other structures are possible. This work compares the cited derivatives as well as some new structures. The criteria used include the ease of synthesis and purification of the derivatized peptide and the effects of the derivative on the peptide sequence fragment ion yield and ionization efficiency. The trimethylammoniumacetyl derivative is concluded to be the most practical for general use, whereas the dimethyloctylammoniumacetyl derivative is found to be desirable for use with hydrophilic peptides. ( Am Soc Mass Spectrom 1995, 6, 428-436)
\end{abstract}

$\mathrm{I}$ $t$ has been observed that the most readily interpretable high energy collision-induced dissociation (CID) spectra are those with a single arginine located at or near a terminus of the peptide [1, 2]. Because the arginine residue strongly favors protonation, the fragment ions are produced by charge-remote fragmentation $[1,3-5]$, which results in predominantly $a_{n}$ and $d_{n}$ ions for peptides with an $N$-terminal arginine and $v_{n}$ and $w_{n}$ ions for those with a C-terminal arginine. The pattern of fragment ions is thus simplified because only two ion series are produced, and these ion series are observed to be complete in most cases. However, a peptide with the less basic lysine in its terminal position will produce both $\mathrm{N}$ - and $\mathrm{C}$ terminal ions of several types. For example, the CID spectrum of the peptide VHLTPVEK contains the following ions: $a_{2}, b_{2}, d_{3}, a_{3}, b_{3}, c_{3}, b_{4}, a_{5}, b_{7}, d_{7}, a_{7}$, and $y_{4}, w a_{5}, z_{5}, y_{5}, w_{6}, x_{6}, x_{7}, v_{8}, w_{8}, z_{8}$ [6]. The result is a spectrum that is less coherent than one with a terminal arginine. Coherent spectra are the most likely to be interpretable in cases where the signal-to-noise ratio is low.

The effect of an arginine residue can be mimicked by derivatizing the terminal residue with a fixed positive charge-carrying molecule. To date, quaternary ammonium [1, 2, 7-9] and phosphonium [10] groups have

\footnotetext{
Address reprint requests to Dr. Klaus Biemann, Department of Chemistry, Room 18-587, Massachusetts Institute of Technology, 77 Massachusetts Avenue, Cambridge, MA 02139-4307.

- Present address: Structural Biology Center, Department of Chemistry \& Biochemistry, University of Maryland-Baltimore County, Baltimore, MD 21228.
}

been used to derivatize peptide termini. The derivatized peptide is desorbed as a fixed-charge cation rather than as an $[\mathrm{M}+\mathrm{H}]^{+}$with a potentially mobile proton. All product ions are in theory produced by chargeremote fragmentation directed by the fixed-charge group [3]. The product ions are predominantly $a_{n}$ and $d_{n}$ for $N$-terminal fixed-charge derivatives and $v_{n}$ and $w_{n}$ ions for C-terminal derivatives $[1,2]$. One of the benefits of this derivatization is that the $d_{n}$ and $w_{n}$ ions produced are useful in differentiating between leucine and isoleucine.

To date, no comparison of charged derivatives for CID spectra has been published. Such a comparison is the purpose of this paper. The present work has been limited to $\mathrm{N}$-terminal derivatives because it is difficult to prepare $C$-terminal derivatives without simultaneous alkylation of the carboxyl group of the side chains of aspartic and glutamic acid. Under proper $\mathrm{pH}$ control, specific $N$-terminal derivatization can be accomplished without also modifying the $\epsilon$-amino groups of lysine [7].

A useful $N$-terminal charged derivative must have several characteristics.

1. The fragment ions must be dominated by $a_{n}$ and $d_{n}$ ions with few gaps, if any, in the product ion pattern.

2. The derivatized peptide must not lose the charged moiety as a neutral fragment to an appreciable extent because fragment ions that lack the derivative moiety would complicate the interpretation of the spectrum. 
3. Derivatization must not adversely affect the ionization efficiency of the peptide.

4. The synthetic step must proceed rapidly and be specific for the $N$ terminus.

Because the major purpose of this work was the assessment of the various $N$-terminal derivatives to define which one has the best mass spectrometric characteristics, the question of yield and applicability to the picomole level has been deferred. Thus, all the reactions were carried out on relatively large samples (50 $\mathrm{nmol}$ ) to produce measurable amounts of each derivative and to avoid that low chemical yield be mistaken for low ionization efficiency. As it turns out, the mass spectrometrically most useful derivative is the one that carries the trimethylammoniumacetyl (TMAA) group at the $N$ terminus. It has already been shown that this derivative can be prepared at the 50-100-pmol level [11].

\section{Materials and Methods}

Peptides were purchased from Sigma Chemical Co. (St. Louis, MO). Dimethyloctylamine, methyl iodide, pyridine, pyridine-4-acetic acid, pyridine-3-acetic acid, dry tetrahydrofuran (THF), and trimethylamine (25-wt \% aqueous solution) were purchased from Aldrich Chemical Co. (Milwaukee, WI); acetic acid, $\gamma$-aminobutyric acid, ammonium bicarbonate, 1-ethyl-3-(dimethylaminopropyl)carbodiimide hydrochloride (EDC), iodoacetic anhydride, and 2-( $\mathrm{N}$-morpholinolethanesulfonic acid buffer from Sigma Chemical Co.; 2,4,6-trimethylpyrilium tetrafluoroborate from Alfa Products (Ward Hill, MA); vinyltriphenylphosphonium bromide from Lancaster Synthesis Inc. (Windham, NH); glass-distilled ethylacetate from EM Science (Gibbstown, NJ); analytical grade chloroform from Mallinkrodt Specialty Chemicals Co. (Paris, KY); high-performance liquid chromatography (HPLC) grade acetonitrile from J.T. Baker Inc. (Philipsburg, NJ); dimethylsulfoxide (DMSO) from Pierce Chemical Co. (Rockford, IL), and anhydrous glycerol and thioglycerol from Fluka Chemika-Biochemika (Ronkonkoma, NY). All peptides and chemicals were used without further purification.

\section{Preparation of Derivatives}

The derivatives (see Figure 1) were prepared in accordance with published procedures or variations thereof. A relatively large amount of peptide $(50 \mathrm{nmol})$ was used so that sufficient peptide derivative was available for the various experiments and comparisons.

Triphenylphosphoniummethyl (TPPE) Derivatives. The published method of derivatization was used: substitution of vinyltriphenylphosphonium bromide for 2bromoethyltriphenylphonium bromide [10]. The derivatized peptides were desalted on a short re-

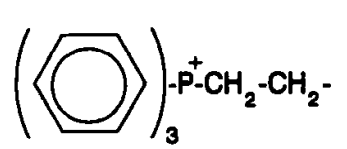

triphenylphosphonlumethyl (TPPE)

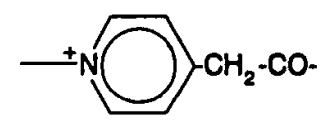

$N$-methyl-4-pyridiniumacetyl (4-NMPA)

$$
\left(\mathrm{CH}_{3}\right)_{3} \mathrm{~N}^{+}-\mathrm{CH}_{2}-\mathrm{CO}
$$

trimethylammoniumacetyl (TMAA)

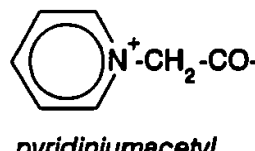

pyridiniumacetyl (PyrA) trimethylpyridiniumbutyl (TMPB)

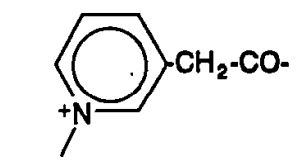

N-methyl-3-pyridiniumacetyl (3-NMPA)

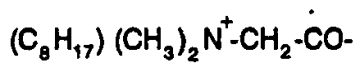

dimethyloctylammoniumacetyl (DMOAA)

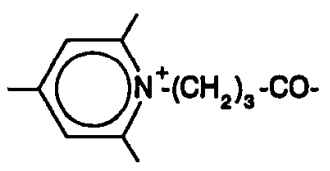

Figure 1. Structures of the fixed-charge derivatives studied.

versed-phase (RP) HPLC column by using a gradient of water and acetonitrile with trifluoroacetic acid (TFA). The eluted fraction was dried by a centrifugal evaporator and dissolved in water on the day the data were acquired.

Trimethylammoniumacetyl (TMAA), dimethyloctylammoniumacetyl (DMOAA), and pyridiniumacetyl (PyrA) derivatives. Peptides were first $\mathrm{N}$-terminally iodoacetylated via an adapted literature procedure [7]. As mentioned before, this method has been used previously to prepare derivatives at the 50-100-pmol level [11]. Unlike the vapor phase procedures [2], derivatization in the liquid phase allows $\mathrm{pH}$ control for selective modification of the $N$ terminus in the presence of lysine [7]. Furthermore, the vapor phase method lends itself only to the derivatization of very small amounts of material, which can be deposited as a very thin layer. This makes it difficult to completely react a complex mixture such as an unfractionated complete enzymatic hydrolyzate of a protein in a single experiment.

For the purpose of this work, the peptide $(50 \mathrm{rmol}$ used to prepare the derivatives for the spectra shown in Figures 2 and 3; $10 \mathrm{nmol}$ to acquire the data compiled in Figure 4) was dissolved in $250-\mu \mathrm{L}$ aqueous $0.1-\mathrm{M}$ sodium acetate-acetic acid buffer ( $\mathrm{pH} 4.0$ ):acetonitrile $1: 1$ and then placed into a conical polypropylene tube, under argon, with a magnetic stirring vane. The solution was chilled on ice and iodoacetic anhydride ( $0.4 \mathrm{M}$ in dry THF; total of $10 \%$ by volume) was added 
a

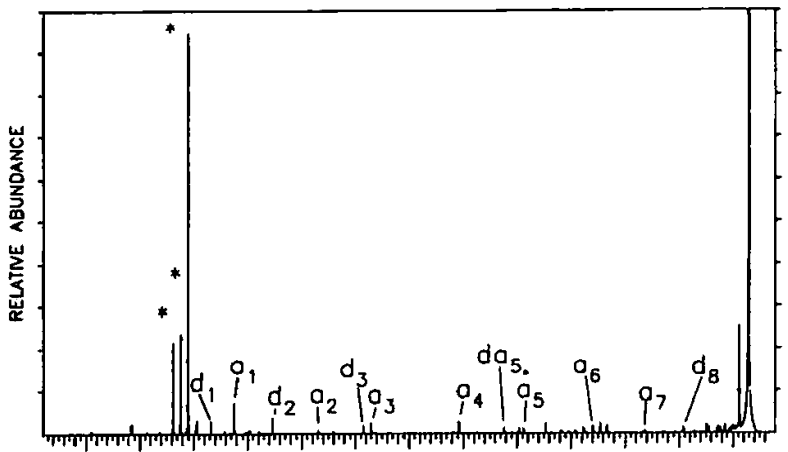

$\begin{array}{lllllllllllll}100 & 200 & 300 & 400 & 500 & 600 & 700 & 800 & 900 & 1000 & 1100 & 1200 & 1300\end{array}$

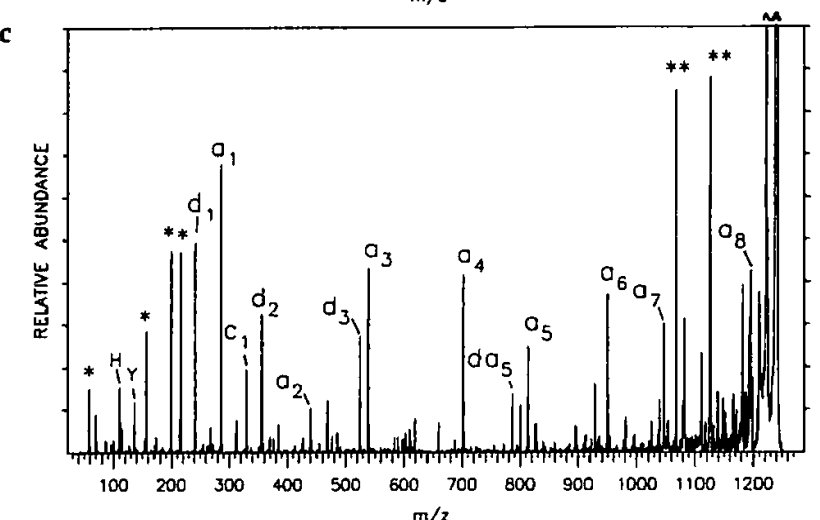

e

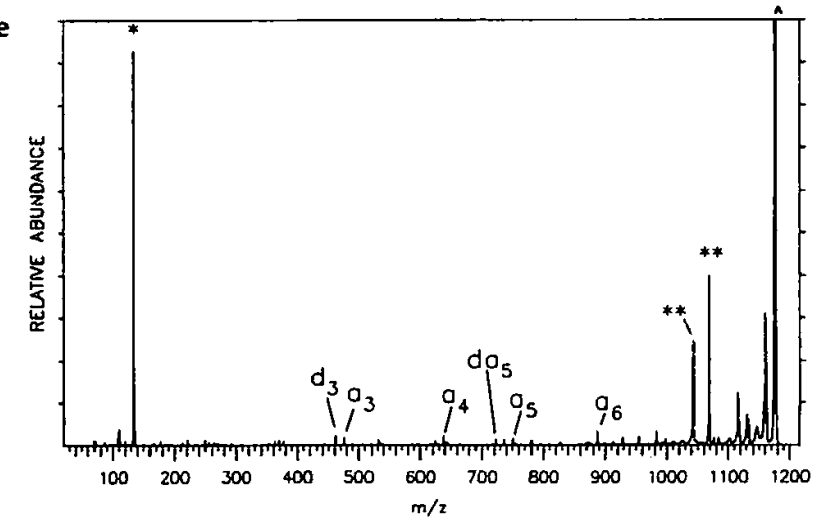

b

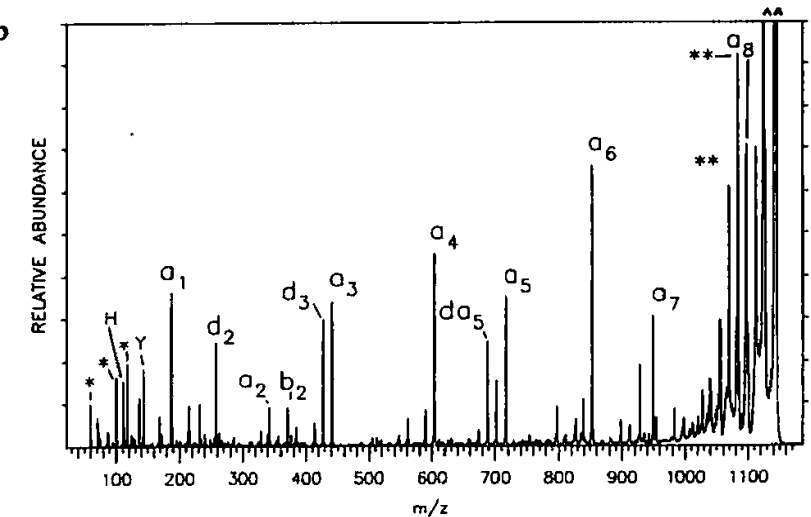

d

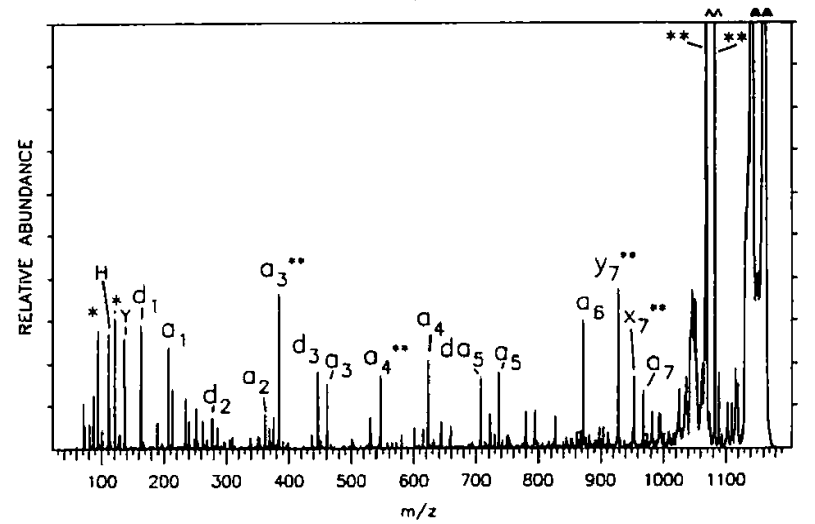

f

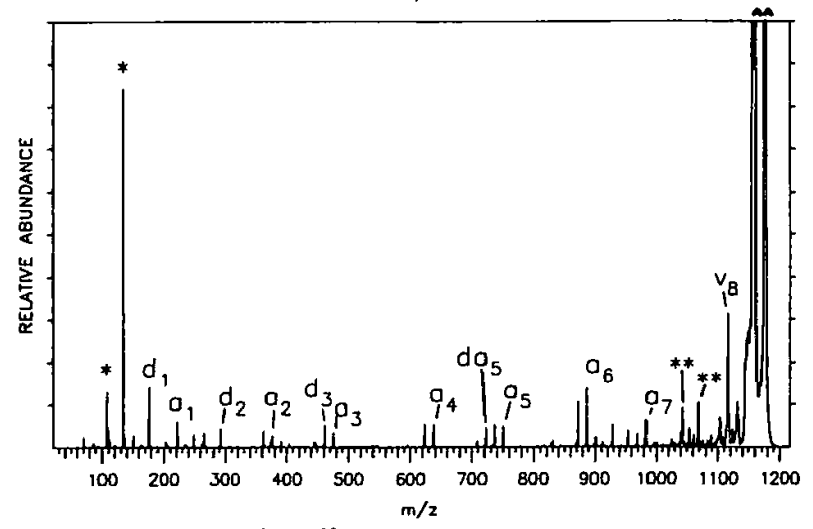

g

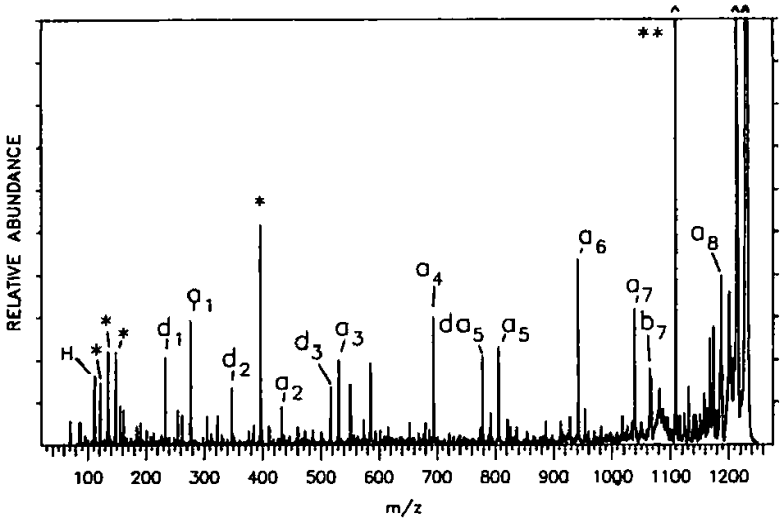

Figure 2. $N$-terminal charged derivatives of the peptide DRVYIHPF: (a) TPPE-DRVYIHPF, $\left[\mathrm{M}^{+}=m / z \text { 1334.1; (b) TMAA-DRVYIHPF, [M }\right]^{+}=m / z$ 1145.1; (c) DMOAA-DRVYIHPF [M $]^{+}=m / z$ 1243.6; (d) PyrA-DRVYIHPF, [M] ${ }^{+}=m / z 1165.7$ (the relative abundance of the $y_{7}^{* *}$ ion is 0.012 ; those of the ions denoted $* *$ are 0.209 and 0.037 , respectively, with increasing $\mathrm{m} / \mathrm{z}$ ); (e) 4-NMPA-DRVYIHPF, [M] ${ }^{+}=m / z$ 1179.4; (f) 3-NMPA-DRVYIHPF, $[\mathrm{M}]^{+}=m / z$ 1179.3; (g) TMPB-DRVYIHPF, [M] ${ }^{+}=m / z 1235.2$ (the relative abundance of the $a_{6}$ ion is 0.021 ; that of the ion denoted ** is 0.061 ). Ions marked with an asterisk contain only the derivative moiety; those marked with a double asterisk have lost part or all of the derivative as a neutral fragment. 

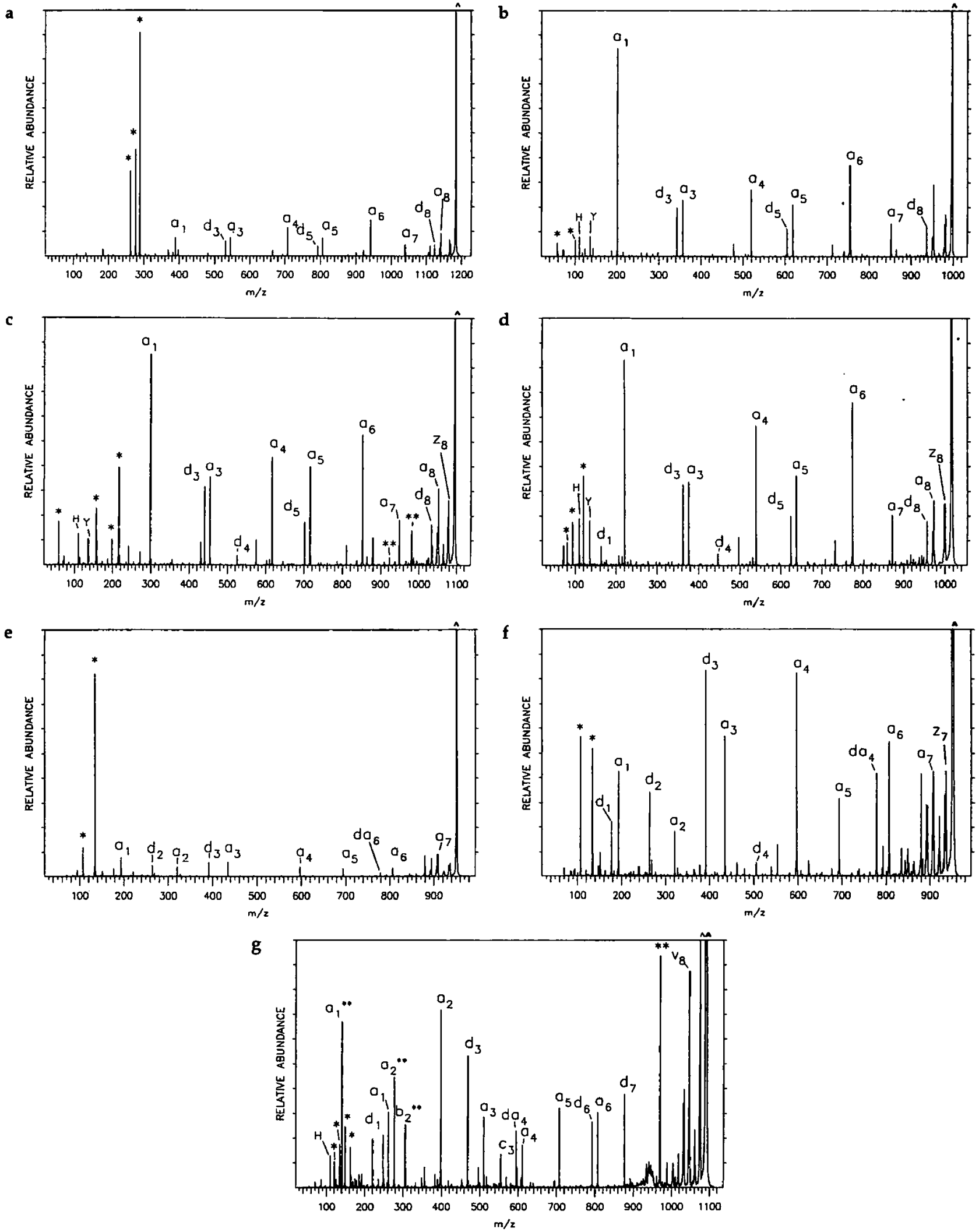

Figure 3. N-terminal charged derivatives of peptides that lack arginine: (a) TPPE-EGVYVHPV, $\left[\mathrm{M}^{+}=m / z\right.$ 1187.5; (b) TMAA-EGVYVHPV, $[\mathrm{M}]^{+}=m / z$ 998.6; (c) DMOAA-EGVYVHPV, [M] ${ }^{+}=$ $m / z$ 1096.5; (d) PyrA-EGVYVHPV, [M] ${ }^{+}=m / z$ 1017.7; (e) 4-NMPA-SONYPIV, [M] ${ }^{+}=m / z$ 953.3; (f) 3-NMPA-SQNYPIV, [M] ${ }^{+}=m / z$ 953.0; (g) TMPB-VHLTPVEK, $\mathrm{E}$ and $\mathrm{K}$ side chains cyclized $[\mathrm{M}]^{+}=m / z$ 1093.1. Ions marked with an asterisk contain only the derivative moiety; those marked with a double asterisk have lost part or all of the derivative as a neutral fragment. 


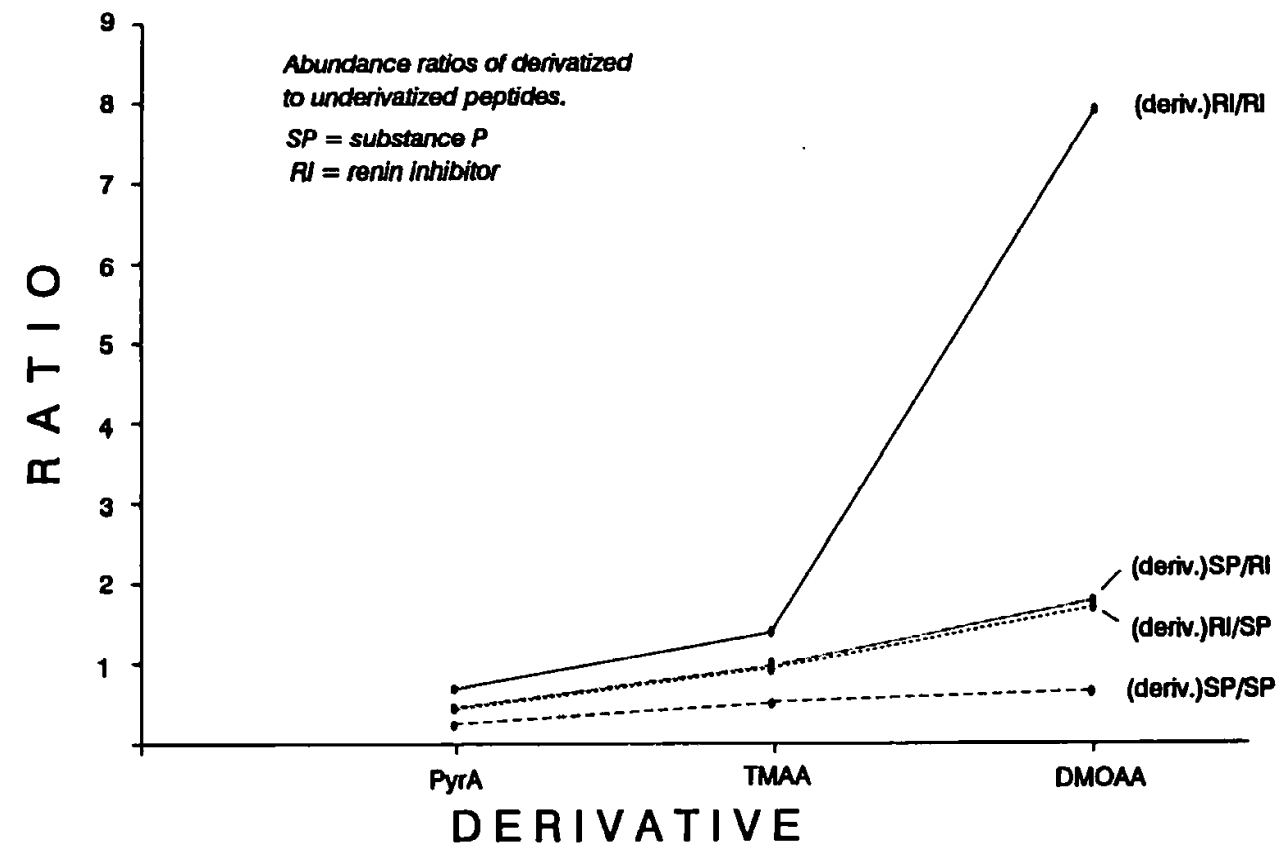

Figure 4. Ratio of abundances of derivatized and underivatized peptides. The peptides used were substance P (RPKPQQFFGLM-NH2) (SP) and renin inhibitor (PHPFHFFFVYK) (RI).

via a syringe through the lid of the polypropylene tube in small aliquots over a 30-min period. The TMAA, DMOAA, and PyrA derivatives were synthesized by adjusting the $\mathrm{pH}$ of the iodoacetylated peptide to 8.5 with ammonium bicarbonate and adding $0.25 \%$ by volume of the appropriate nucleophile (trimethylamine, dimethyloctylamine, or pyridine, respectively). The mixture was incubated at $37^{\circ} \mathrm{C}$ for $1 \mathrm{~h}$, acidified with acetic acid ( $1 \%$ by volume), desalted on a RPHPLC column, and dried by a centrifugal evaporator. Tetrahydrothiophene can be used as the nucleophile in the last step to synthesize tetrahydrothiophenium derivatives by the same procedure.

$N$-methyl-4-pyridiniumacetyl (4-NMPA) derivatives. Peptide ( $50 \mathrm{nmol} ; 1 \mathrm{nmol} / \mu \mathrm{L}, \mathrm{H}_{2} \mathrm{O}$ ) was mixed with $50-\mu$ L pyridine-4-acetic acid $\left(1 \mathrm{M}, \mathrm{H}_{2} \mathrm{O}\right)$, to which solution was added $50-\mu \mathrm{L}$ EDC $\left(0.4 \mathrm{M}, \mathrm{H}_{2} \mathrm{O}\right)$. The solution was adjusted to $\mathrm{pH} 4.0$ with $1-\mathrm{M} \mathrm{NaOH}$. The reaction proceeded for $2 \mathrm{~h}$ at room temperature. The solution was dried in a centrifugal evaporator and redissolved in 200- $\mu \mathrm{L}$ DMSO. Methyliodide (5 $\mu \mathrm{L}$ ) was added and the solution was incubated under argon overnight at $37^{\circ} \mathrm{C}$ in the dark. The solution was extracted with ethylacetate followed by chloroform and reduced to a volume of $\sim 30 \mu \mathrm{L}$ in a centrifugal evaporator. The solution was diluted with 200- $\mu \mathrm{L} 50 \%$ acetic acid, filtered, desalted by using a short RP-HPLC column, and dried in a centrifugal evaporator. The sample was dissolved in water on the day the mass spectra were acquired. The yield was low but sufficient for the acquisition of CID tandem mass spectra.
$N$-methyl-3-pyridiniumacetyl (3-NMPA) derivatives. The procedure was essentially the same as for 4-NMPA, but pyridine-3-acetic acid was used as the reagent.

Trimethylpyridiniumbutyl (TMPB) derivatives. A published method was adapted for the synthesis of the derivatization reagent [12]. $\gamma$-Aminobutyric acid (1.0 $\times$ $10^{-3} \mathrm{~mol}$ ) and 2,4,6-trimethylpyrilium tetrafluoroborate $\left(1.5 \times 10^{-3} \mathrm{~mol}\right)$ were mixed with $5-\mathrm{mL} \mathrm{H}_{2} \mathrm{O}$. The $\mathrm{pH}$ of the mixture was adjusted to 9.0 by dropwise addition of $1-\mathrm{N} \mathrm{NaOH}$. The reaction was allowed to proceed for $48 \mathrm{~h}$ at room temperature, adjusting the $\mathrm{pH}$ to 9.0 with $1-\mathrm{N} \mathrm{NaOH}$ as needed. The resulting solution was acidified with acetic acid and used to derivatize peptides.

The peptide (50 nmol; $1 \mathrm{nmol} / \mu \mathrm{L}$ in $\mathrm{H}_{2} \mathrm{O}$ ) was mixed with $50-\mu \mathrm{L}$ dry THF, $50-\mu \mathrm{L}$ of the solution, and $30-\mu \mathrm{L} 1 \% \mathrm{HCl}$, to which was added $50-\mu \mathrm{L} 1-\mathrm{M}$ EDC (final $\mathrm{pH} 4.5$ ). The reaction proceeded for $2 \mathrm{~h}$ at room temperature, after which time the solution was extracted with ether. The volume of the solution was reduced by half by using a centrifugal evaporator. The solution was filtered, desalted on a short RP-HPLC column, and dried. The fraction was dissolved in water on the day mass spectra were acquired. The yield of TMPB-derivatized peptides was low but sufficient for determination of CID characteristics of these derivatives.

\section{Fast-Atom Bombardment Mass Spectrometry}

Fast-atom bombardment mass spectrometry (FAB-MS) of peptides, dissolved in glycerol or thioglycerol, was 
carried out in the first (MS-l) of two mass spectrometers of a tandem high resolution mass spectrometer (JEOL HX110/HX110; JEOL, Tokyo, Japan) at an accelerating voltage of $10 \mathrm{kV}$ and a mass resolution of 1:2600 (10\% valley). Single scans were acquired at a scan rate from $m / z 0$ to $m / z 6000$ in $1 \mathrm{~min}$ (corresponding to $0.8 \mathrm{~min}$ for $\mathrm{m} / \mathrm{z} 200-2000$ ). For calibration, (CsI) ${ }_{n} \mathrm{Cs}^{+}$cluster ions were used. The JEOL Cs ${ }^{+}$ ion gun was operated at $26 \mathrm{kV}$. Mass spectra were acquired with a JEOL DA5000/6000 data system running on a DEC PDP $11 / 73$ computer (Digital Equipment Corp., Maynard, MA).

\section{Tandem Mass Spectrometry}

Tandem mass spectrometry was carried out by using all four sectors of the JEOL HX110/HX110, an instrument of $E_{1} B_{1} E_{2} B_{2}$ configuration. CID of protonated peptide molecules, selected by MS-1, took place in the field-free region after $B_{1}$; thus both MS-1 and MS-2 were operated as double-focusing instruments. The collision cell potential was held at $3.0 \mathrm{kV}$; therefore, the ion collision energies were at $7.0 \mathrm{keV}$. The collision gas (helium) was introduced into the collision chamber at a pressure sufficient to reduce the precursor ion signal by $65-70 \%$. The CID mass spectra were recorded with $100-\mathrm{Hz}$ filtering, scanning from $\mathrm{m} / \mathrm{z} 50$ to $\mathrm{m} / \mathrm{z}$ 1950 in 1 min. MS-1 was operated at a resolution adjusted so that only the ${ }^{12} \mathrm{C}$ species of the protonated peptide molecule to be analyzed was transmitted. MS-2 was operated at a resolution of 1:1000, and was calibrated with a mixture of CsI, $\mathrm{NaI}, \mathrm{KI}, \mathrm{RbI}$, and $\mathrm{LiCl}$ [13].

\section{Results and Discussion}

The structures of the charged groups attached to the $N$-terminal amino acid of the peptides studied are shown in Figure 1 [14]. The TMAA, DMOAA [2], and TPPE [10] derivatives have been described previously. The TMPB derivative is a modification of a lysine specific derivative used in earlier work in this laboratory [1]. The 4-NMPA and 3-NMPA derivatives have not been used before and the PyrA is a structure related to the aliphatic quaternary ammonium derivatives. It should be pointed out that the positive "molecular" ion observed in the FAB spectrum of the salt consisting of a quaternary ammonium ion and an anion is the cation itself. For the purpose of simplicity, this precursor ion will be denoted $[\mathrm{M}]^{+}$in the following text and in the figures.

\section{General Characteristics of the Charged Derivatives}

In the course of this work, six peptides were used to test the effects of each modifying group. It was observed in each case that the most important factor that affects the CID spectrum for a given derivative was the presence of arginine. Accordingly, Figure 2 shows the peptide DRVYIHPF modified at its $N$-terminal amino group with the substituents shown in Figure 1. This peptide illustrates clearly that arginine seems to be able to abstract a proton for the charged moiety and eliminate part or all of it as a neutral fragment. This produces additional ions that may render the interpretation of the spectrum more difficult. The extent of this elimination depends on the nature of the derivative. Figure 3 shows the effect of charged derivatization on peptides that lack arginine. Figure 2 exhibits the CID spectra of the same peptide, DRVYIHPF, modified by all of the substituents shown in Figure 1, whereas Figure 3 illustrates the spectra of the derivatives of peptides that do not contain arginine. The spectra are plotted with a single magnification factor so as to keep most peptide fragment ions on scale. Ions marked with an asterisk contain only the derivative moiety; those marked with a double asterisk have lost part. or all of the derivative as a neutral fragment.

To provide a realistic display that demonstrates the relative contribution of ion current carried by the fragments related to the quaternary substituent (or its loss from the precursor ion) compared to that of the sequence characteristic fragment ions, all spectra are plotted in such a way that the major fragment ion, whatever its source, is at or near full scale (exceptions are indicated in the figure legends).

The CID spectra of (TPPE)-DRVYIHPF and -EGVYVHPV (angiotensin II antipeptide) are shown in Figures $2 a$ and $3 a$, respectively. These spectra are dorninated by ions at $m / z 262,277$, and 289 (labeled with asterisks), which are solely produced from the derivative moiety and thus contain no sequence information. The peptide fragment ions are of rather low relative abundance in both spectra, although this appearance is deceiving as will be shown in the discussion of fragment ion yield. The signal-to-noise ratio is, however, very high, and magnification of the $Y$ axis clearly reveals a complete series of $a_{n}$ and $d_{n}$ ions.

The CID spectra of (TMAA)-DRVYIHPF and -EGVYVHPV are shown in Figures $2 b$ and $3 b$, respectively. The low mass ions produced from the derivatizing group $(m / z 58,100$, and 117) are quite low in abundance and the expected $a_{n}$ and $d_{n}$ ions are observed. Ions at $[M-59]^{+}$and $[M-73]^{+}(* *)$ are observed in the spectrum of (TMAA)-DRVYIHPF as well as other arginine-containing peptides [6] and are absent for peptides that lack arginine (for example, Figure $3 \mathrm{~b}$ ). The elimination of part or all of the derivative moiety as a neutral fragment for TMAA as well as DMOAA and PyrA derivatives is believed to be caused by the arginine side chain, either by extraction of a proton or by direct displacement, similar to the mechanism shown in Scheme I, which will be described in the next paragraph. These ions are not sufficiently abundant to detract from the quality of the spectrum. The same peptides derivatized with DMOAA (Figures $2 c$ and $3 c$ ) and PyrA (Figures $2 d$ and $3 d$ ) exhibit similar fragmentation patterns, although the ions pro- 


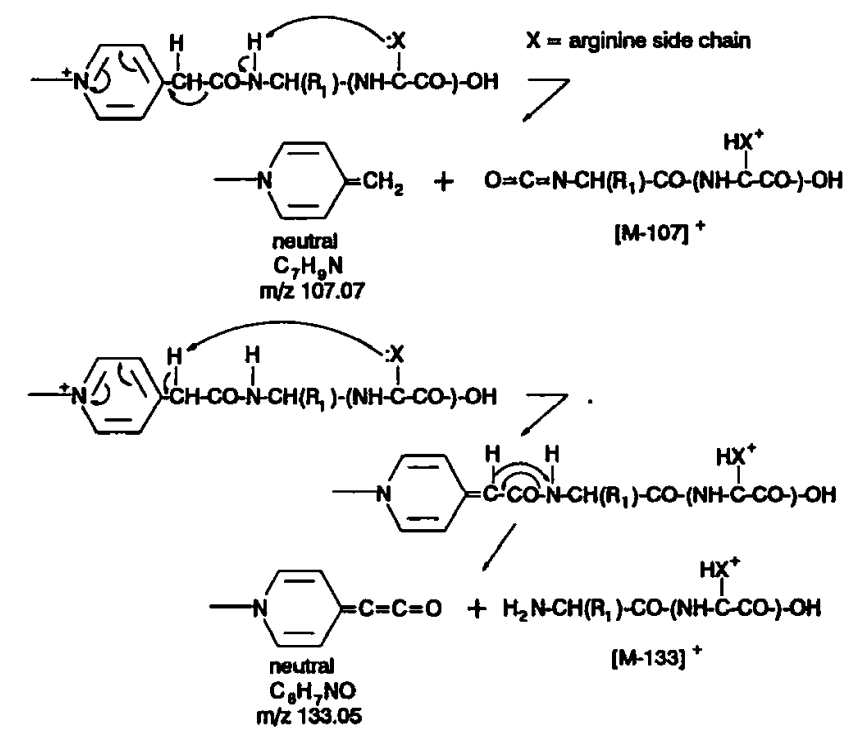

Scheme I. Mechanisms for the formation of $[M-107]^{+}$and $[M-133]^{+}$ions from 4NMPA derivatized precursor ions.

duced from the derivative moiety at low and high mass are somewhat more abundant than those observed for the TMAA derivative. The $a_{3}^{* *}$ and $a_{4}^{* *}$ ions in Figure $2 \mathrm{~d}$ have lost part of the derivative moiety, and the $y_{7}^{* *}$ and $x_{7}^{* *}$ ions correspond to fragments that have lost the derivatizing moiety.

The 4-NMPA derivative produces ions due to fragmentation of derivative-peptide bonds that are very abundant relative to the peptide fragment ions as shown for DRVYIHPF and SQNYPIV in Figures $2 e$ and 3 e, respectively. The former spectrum shows abundant $[\mathrm{M}-107]^{+}$and $[\mathrm{M}-133]^{+}$ions, which are absent in the spectrum of the latter. It is believed that these losses are produced when arginine extracts a proton, which eliminates the derivative moiety as shown in Scheme I. Also present in Figure $2 \mathrm{e}$ are $\mathrm{N}$-terminal ions that lack the derivative moiety that corresponds to $d_{1}^{* *}, a_{1}^{* *}, b_{1}^{* *}, d_{2}^{* *}$, and $a_{2}^{* *}$ (in low abundance and therefore not labeled) presumably produced from the $[\mathrm{M}-133]^{+}$ion. The 3-NMPA-DRVYIHPF derivative, in contrast, produces more abundant peptide fragmentation and less abundant $[\mathrm{M}-107]^{+}$and $[\mathrm{M}-133]^{+}$ ions, as shown in Figure 2f. The spectrum of 3NMPA-SQNYPIV (Figure 3f) shows that the ions of $m / z 107$ and 134 produced from the derivative are less abundant relative to the peptide fragment ions than observed for the 4-NMPA derivative of the same peptide (Figure 3e). It would therefore appear that elimination of the derivative is less favored for the 3-NMPA derivative than for 4-NMPA. A possible explanation is that the pyridinium nitrogen is para to the acetyl linkage in the 4-NMPA and meta in the 3-NMPA derivative. The former is able to transfer charge via resonance to the peptide, which allows the derivative moiety to leave as a neutral; the latter cannot transfer charge via resonance, which necessitates its elimination as a zwitterion, evidently a less favored process.
The spectra of TMPB-derivatized DRVYIHPF and VHLTPVEK are shown in Figures $2 \mathrm{~g}$ and $3 g$, respectively. The ions produced from the derivative moiety $(m / z 121,135,148$, and 162 , labeled with an asterisk) are of lower relative abundance compared to those of the other derivatives. The expected $a_{n}$ and $d_{n}$ ions are observed for both TMPB peptides. It is notable, in Figure $3 \mathrm{~g}$, that as would be expected the $c_{3}$ ion is observed to precede a threonine residue [15]. All ions marked with a double asterisk in Figures $2 \mathrm{~g}$ and $3 g$ have lost trimethylpyridine as a neutral moiety $(121 \mathrm{u})$. These ions are observed for many of the TMPBderivatized peptides and increasingly complicate these spectra. The abundant ion at $m / z 397$ (marked with an asterisk) in the spectrum of TMPB-DRVYIHPF (Figure $2 \mathrm{~g}$ ) is characteristic of this derivative in the presence of arginine.

\section{Effect of Charged Derivatization on Fragment Ion Yield}

According to the literature, TMAA $[2,11]$ and TPPE [10] are the two most frequently used fixed-charge derivatives for peptides, although their spectra are quite different in appearance. The TMAA derivative produces ions of very low abundance from the loss of the derivative moiety relative to the peptide fragment ions, whereas those from TPPE are quite high in relative abundance. To directly compare the effects these derivatives have on the quality of the CID spectra, the peptide fragment ion yields for derivatized versus underivatized peptides were measured for the peptides EGVYVHPV and SQNYPIV by using the TMAA and TPPE derivatives. The yield was determined by normalizing the peptide sequence fragment ion abundances to the precursor ion abundance (average taken before and after the CID spectrum was acquired) and averaging the normalized abundances. The results are shown in Table 1 for measurements made on three separate days. The peptide fragment ion yield is clearly improved relative to the underivatized analog for these two peptides by the use of either charged derivative. It

Table 1. Efficiency of peptide sequence fragment ion formation for derivatized and underivatized peptides

\begin{tabular}{llccc}
\hline & & \multicolumn{3}{c}{ Fragment Ion Yields $^{\circ}$} \\
\cline { 3 - 5 } \multicolumn{1}{c}{ Peptide } & & Unalkylated & TMAA & TPPE \\
EGVYVHPV & Day 1 & 1.89 & 4.72 & 2.40 \\
& Day 2 & 0.87 & 3.47 & 7.96 \\
\multirow{4}{*}{ SONYPIV } & Day 3 & 1.67 & 5.28 & 8.60 \\
& Day 1 & 1.68 & 2.84 & 2.40 \\
& Day 2 & 2.56 & 2.04 & 4.52 \\
& Day 3 & 2.14 & 4.57 & 5.57 \\
\hline
\end{tabular}

\footnotetext{
The peptide sequence fragment ion abundances were normatized to the abundance of the precursor ion measured at the detector situated after MS-1 (average taken before and after the CID scan). The normalized abundances were averaged, excluding immonium ions and ions produced from amino acid side chain losses, to give the values shown.
} 
is not clear, however, from these data which derivative produces the higher yield of peptide fragment ions. It can be concluded, however, that the high abundance ions produced from the TPPE derivative moiety do not adversely affect the yield of peptide sequence product ions. Therefore, given the same precursor ion abundance, peptides derivatized with either of the derivatives tested should yield peptide sequence fragment ions of approximately the same abundance.

\section{Ease of Derivatization}

The synthesis of the 3-NMPA, 4-NMPA, and TMPB derivatives is accomplished by the use of EDC coupling. This method has not been found to produce sufficiently high yields for general use. The TMAA, DMOAA, and PyrA derivatives are synthesized by treatment of the $\mathrm{N}$-terminal iodoacetylated peptide with the appropriate nucleophile. The iodoacetylation occurs in $80-90 \%$ yield and is specific for the $N$ terminus even in the presence of lysine $[7,11]$ for pure peptides. Reaction with the nucleophile also proceeds in high yield. It is important to remove excess reagents from the peptide to avoid adverse effects on the ionization efficiency of the derivatized peptide. This removal can be accomplished with a rapid desalting step for trimethylamine and pyridine (by using a short reversed-phase cartridge and a single step elution), although the more hydrophobic dimethyloctylamine is best removed by using a more time-consuming reversed-phase gradient. The TPPE reagent was found to produce several by-products that are retained on the HPLC column and that co-elute with some derivatized peptides when a reversed-phase gradient fractionation is used. The ionization efficiency of some TPPE-derivatized peptides was found to be quite poor relative to the underivatized peptide, possibly because of suppression by the co-eluting reagent. Also, the synthetic step for TPPE, which requires incubation at $\mathrm{pH} 9$ [10], leads to reaction with the $\epsilon$-amino group of lysine in addition to the $N$ terminus.

\section{Effect of Derivatization on Ionization Efficiency}

The synthesis and/or fragment ion problems discussed in the foregoing text effectively eliminate all but the TMAA, DMOAA, and PyrA derivatives from practical consideration. An experiment was thus conducted to determine the changes in ionization efficiency caused by derivatization with each of the three derivatives. This experiment involved the acquisition of data on a mixture of equimolar amounts of a derivatized and an underivatized peptide in the normal FAB-MS mode. The ratios of the abundances of the derivatized relative to the underivatized peptides are plotted in Figure 4. The two peptides used were substance $\mathrm{P}$ (RPKPQQFFGLM-NH ${ }_{2}$ ) and human renin inhibitor (PHPFHFFFVYK). The results show that the DMOAA derivative ionizes most efficiently among the three derivatives tested. The PyrA derivative has a negative effect on ionization efficiency relative to the underivatized peptide and the effect of TMAA derivatization is close to neutral. This conclusion supports that of another study [11].

To better compare their performance, these three derivatives were prepared for a variety of peptides. Table 2 summarizes the results, which indicate the presence of high mass ions produced from loss of the neutral derivative moiety for each derivative-peptide combination. The results show that TMAA and PyrA produce these high mass ions in the presence of arginine only. The DMOAA derivative produces these ions for all arginine- and some lysine-containing peptides. In addition, DMOAA loses its octyl chain to produce an $[\mathrm{M}-113]^{+}$ion in nearly all spectra. The conservative choice for a derivative for the sequencing of peptides of unknown structure is thus the TMAA group. This derivative produces the desired $a_{n}$ and $d_{n}$ ions, whereas the ions from the derivative moiety are of minimal abundance.

From these examples, it can be concluded that complete or nearly complete sequence information can be deduced from the CID spectrum of a TMAA-derivatized peptide provided that there is sufficient precur-

Table 2. High mass ions that result from the loss of a neutral fragment observed in the CID spectra of peptides derivatized with TMAA, DMOAA, and PyrA, respectively

\begin{tabular}{|c|c|c|c|c|c|c|c|}
\hline \multirow[b]{2}{*}{ Peptide } & \multicolumn{2}{|c|}{ TMAA } & \multicolumn{3}{|c|}{ DMOAA } & \multicolumn{2}{|c|}{ PyrA } \\
\hline & $\overline{[M-59]^{+}}$ & $\overline{[M-73]^{+}}$ & $\overline{[M-113]^{+}}$ & {$[\mathrm{M}-157]^{+}$} & $\overline{[M}-171]^{+}$ & $\overline{[M-79]^{+}}$ & {$\left[\mathrm{M}-\overline{93}^{+}\right.$} \\
\hline $\begin{array}{l}\text { RYLGYLE } \\
\text { DRVYIHPF } \\
\text { PVOVR } \\
\text { HLGLAR } \\
\text { KFIGLM } \\
\text { AKFDKFYGLM } \\
\text { VHLTPVEK } \\
\text { EGVYVHPV } \\
\text { SONYPIV } \\
\text { YGGFL }\end{array}$ & $\dot{\bullet}$ & $\begin{array}{l}\bullet \\
\bullet \\
\bullet\end{array}$ & $\begin{array}{l}\dot{1} \\
: \\
\vdots \\
\vdots \\
\end{array}$ & $\dot{\bullet}$ & $\dot{\bullet}$ & $\ddot{\bullet}$ & $\dot{\bullet}$ \\
\hline
\end{tabular}


sor ion current. The synthetic step, which followed a published method [7], has been found to work in high yield (80-90\%) while it alkylates only the $N$ terminus of pure peptides.

Finally, it should be noted that the CID spectra of the same peptide carrying a fixed positive charge embedded in a different structure, as one may expect, exhibit essentially the same sequence-specific peptide ions (compare Figures $2 a-g, 3 a-d$, and $3 e$ and $f$ ). The main difference is their relative abundance in comparison to that of the fragment ions due to the derivative moiety. To make the relative contributions of these (undesirable) ions clearer, the spectra were plotted in such a way that the major ions are still on scale.

\section{Conclusions}

All tested derivatives direct the production of $a_{n}$ and $d_{n}$ ions in the data presented as well as with other peptides [6]. The derivatives vary in the extent to which they generate ions from loss of the derivative moiety at low and high mass. Losses of part or all of the moiety as a neutral fragment to produce high mass ions is most significant for arginine-containing peptides. Undesirable fragmentations and/or synthetic difficulties eliminate many of the derivatives from practical use. The TPPE derivative produces very abundant low mass ions, although these ions were found to have no negative effect on peptide fragment ion yield. Difficulty in removal of the derivatizing reagent from the peptide and inability to differentiate the $N$ terminus from the $\epsilon$-amino group of lysine eliminates the TPPE derivative as a practical alternative. The 4-NMPA derivative fragmented poorly, especially for arginine-containing peptides. The TMPB and 3-NMPA derivatives performed relatively well, although the EDC coupling did not produce satisfactory yield. The TMAA derivative is found to be best suited for general use because it produces the lowest abundance of ions related to the derivative moiety: its effect on ionization efficiency is neutral and it can be prepared easily for pure peptides. The DMOAA derivative appears to have a positive effect on ionization efficiency, which makes it a possible choice for peptides that ionize poorly, although a reversed-phase gradient fractionation is necessary to remove dimethyloctylamine from the peptide. The PyrA derivative produces a fragment ion profile similar to that of TMAA, although it has a negative effect on ionization efficiency.

\section{Acknowledgments}

The authors appreciate the helpful discussions with Dr. Stephen A. Martin, then at Genetics Institute, Andover, MA. The work was supported by The National Institutes of Health (grants NIEHS ES04675, NIH GM05472, and RR00317).

\section{References}

1. Johnson, R. S.; Martin, S. A.; Biemann, K. Int. J. Mass Spectrom. Ion Processes 1988, 86, 137-154.

2. Vath, J. E.; Biemann, K. Int. J. Mass. Spectrom. Ion Processes 1990, 100, 287-299.

3. Tomer, K. B.; Crow, F. W.; Gross, M. L. I. Am. Chem. Soc. 1983, 105, 5487-5488.

4. Adams, J. Mass Spectrom. Rev. 1990, 9, 141-186.

5. Johnson, R. S.; Martin, S. A.; Biemann, K.; Stults, J. T.; Watson, J. T. Anal. Chem. 1987, 59, 2621-2625.

6. Zaia, J. Ph.D. dissertation, Massachusetts Institute of Technology, Cambridge, MA, 1993.

7. Wetzel, R.; Halualani, R.; Stults, J. T.; Quan, C. Bioconj. Chem. 1990, 2, 114-122.

8. Stults, J. T. Proceedings of the 40th ASMS Conference on Mass Spectrometry and Allied Topics; 1992, pp. 1815-1816.

9. Kidwell, D. A.; Ross, M. M.; Colton, R. J. J. Am. Chem. Soc. 1984, 106, 2219-2220.

10. Wagner, D. S.; Salari, A.; Gage, D. A.; Leykam, J.; Fetter, J.; Hollingsworth, R.; Watson, J. T. Biol. Mass Spectrom. 1991, 20, 419-425.

11. Stults, J. T.; Lai, J.; McCune, S.; Wetzel, R. Anal. Chem. 1993, 65, 1703-1708.

12. O'Leary, M. H.; Samberg, G. A. J. Am. Chem. Soc. 1971, 93, 3530-3532.

13. Sato, K.; Asada, R.; Ishihara, M.; Kunihiro, F.; Kammei, Y.; Kubota, E.; Costello, C. E.; Martin, S. A.; Scoble, H.; Biemann, K. Anal. Chem. 1987, 59, 1652-1659.

14. Zaia, J.; Biemann, K. Proceedings of the 41st ASMS Conference on Mass Spectrometry and Allied Topics; 1993, pp. 358a-358b.

15. Downard, K. M.; Biemann, K. J. Am. Soc. Mass Spectrom. 1993, 4, 874-881. 